\title{
OPA3-Related Autosomal Dominant Optic Atrophy and Cataract with Ataxia and Areflexia
}

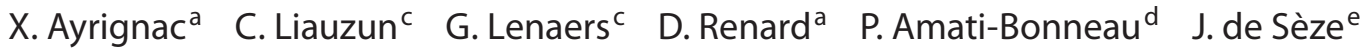 \\ H. Dollfus ${ }^{f} \quad$ C. Hamel $^{\text {b, c }} \quad$ D. Bonneau ${ }^{d} \quad$ P. Labauge ${ }^{a}$ \\ a Department of Neurology and ' ${ }^{b}$ Reference Center 'Affections Sensorielles Génétiques', Ophthalmological \\ Department, Montpellier University Hospital, and ' Montpellier Neurosciences Institute, INSERM U1051, \\ Montpellier, ${ }^{d}$ Biochemistry and Genetic Department, Angers University Hospital and UMR-INSERM, U1083-CNRS, \\ Angers, ${ }^{e}$ Department of Neurology and ${ }^{f}$ Genetic Department, Strasbourg University Hospital, Strasbourg, France
}

Dear Sir,

Hereditary optic atrophies refer to a heterogeneous group of genetic disorders in which the most common form is autosomal dominant optic atrophy (ADOA). Only two genes, i.e. OPA 1 and $O P A 3$, have been identified in ADOA so far [1-3]. Mutations in OPA1 are responsible for 60$80 \%$ of familial cases of ADOA while OPA3 has been implicated in only two families with ADOA and associated cataract (ADOAC) [2, 3]. Additional neurological signs have been reported in about $20 \%$ of OPA1-mutated patients and have also been described in some OPA3-mutated patients [2, 3]. Recessive mutations in OPA3 are responsible for type III 3-methylglutaconic aciduria (the so-called Costeff syndrome) consisting of early-onset bilateral optic atrophy, spasticity, extrapyramidal signs, and cognitive deficit [4]. Here we describe a third family harboring a dominant mutation in OPA3 responsible for ADOAC with additional neurological features.

Ophthalmological signs of this 38-yearold woman consisted of (1) bilateral optic

X. Ayrignac and C. Liauzun contributed equally to this work. atrophy and nystagmus since the first year of life, (2) progressive loss of vision, and (3) bilateral cerulean cataract at age 37 . Additional symptoms consisted of intractable constipation alternating with severe diarrhea since childhood, together with gait unsteadiness, paresthesias in the four extremities, cramps, and burning pain in the lower limbs since the age of 35 . Clinical examination at the age of 38 showed cerebellar ataxia, lower limb areflexia, pinprick and light-touch hypoesthesia, and pes cavus. Ophthalmological examination showed a visual acuity of 1/10 in both eyes. Eye fundus examination disclosed bilateral optic atrophy. Goldmann visual field examination revealed bilateral central scotoma, and slit-lamp examination showed bilateral cerulean cataract. Electroretinography was normal and visual-evoked potentials showed bilateral optic nerve dysfunction. Motor as well as sensory nerve conduction studies, myography, and motor-evoked potentials were all normal. Somatosensory-evoked potentials disclosed prolonged latencies of cortical as well as lumbar potentials. Brain MRI only showed mild cerebellar atrophy and MR spectroscopy was normal. Blood tests including serum lactate and pyruvate concentrations were normal.
The proband's 39-year-old brother had bilateral optic atrophy without additional neurological symptoms and her 63-yearold mother showed bilateral atrophy optic, nystagmus, generalized areflexia, and severe decrease of vibration sense in the lower limbs.

In all affected patients, the ten primary mtDNA mutations causing Leber hereditary optic neuropathy were excluded by direct sequencing. The direct sequencing of coding exons and exon-intron boundaries of OPA3 evidenced the heterozygous c.313C $>$ G (p.Q105E) mutation in exon 2 in all 3 affected patients. Analysis of the proband's fibroblasts using immunohistochemistry and specific antibodies raised against the peptides corresponding to $O P A 3$ exon 2 and exon $2 \mathrm{~b}$ revealed uniform mitochondrial localization of both OPA3 isoforms, a normal reticulated mitochondrial network and fluorescence labeling intensities similar to those found in age- and sex-matched control fibroblasts (fig. 1a). Quantifications of OPA3 mRNA variants showed a global increase in the expression of the OPA3 variant 1 , supporting a dominant negative effect of the mutation (fig. 1b). Treatment with two different apoptotic drugs further substantiates a deficit in cell survival in patient cells (fig. 1c).

\section{KARGER}

Fax +4161306 1234 E-Mail karger@karger.ch www.karger.com
(C) 2012 S. Karger AG, Basel 0014-3022/12/0682-0108\$38.00/0

Accessible online at: www.karger.com/ene
Dr. Xavier Ayrignac

Service de Neurologie

CHU Gui de Chauliac, 80 Ave. Augustin Fliche

FR-34295 Montpellier (France)

Tel.+33 4673374 13, E-Mail xavier.ayrignac@yahoo.fr 
Fig. 1. a Immunofluorescence imaging of the fibroblasts using OPA3-specific antibodies. Fluorescent pictures of control and patient fibroblasts using the ATP synthase antibody (top and bottom, left) OPA3 isoform 1 (top, middle) and OPA3 isoform 2 (bottom, middle) antibodies, and their merged representation with Hoechst staining (top and bottom, right) reveal a uniform labeling of the mitochondrial network, which appears tubulated in control and patient fibroblasts (bars represent $20 \mu \mathrm{m})$. $\mathbf{b}$ Assessment of the abundance of $O P A 3$ mRNA variants in control and patient fibroblasts. Results from real-time PCR analysis were obtained using a couple of primers specific to exon 2 and exon $2 b$, specific to $O P A 3$ variant 1 (var. 1 , left) and variant 2 (var. 1, right) on total cDNA from control (Cont.) and patient (Pat.) fibroblasts. Mean relative amounts were calculated on a basis of 100 for the OPA3-var. 1 in control fibroblasts. c Assessment of the survival ratio of control and patient fibroblasts in the presence of apoptotic stimuli. Relative percentage of cells surviving after the treatment of control and patient fibroblast cultures with staurosporine (STS, 0.5 $\mu \mathrm{M})$ for $3 \mathrm{~h}$, and lonidamine (LND, $0.5 \mu \mathrm{M}$ ) for $24 \mathrm{~h}$. Control cells were arbitrarily assigned a value of $100 \%$. All data are mean $\pm \mathrm{SD}$ of results from at least separated experiments reproduced in duplicates.
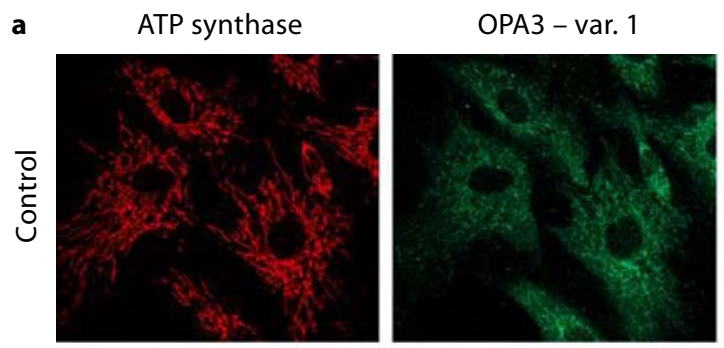

Merge + Hoechst
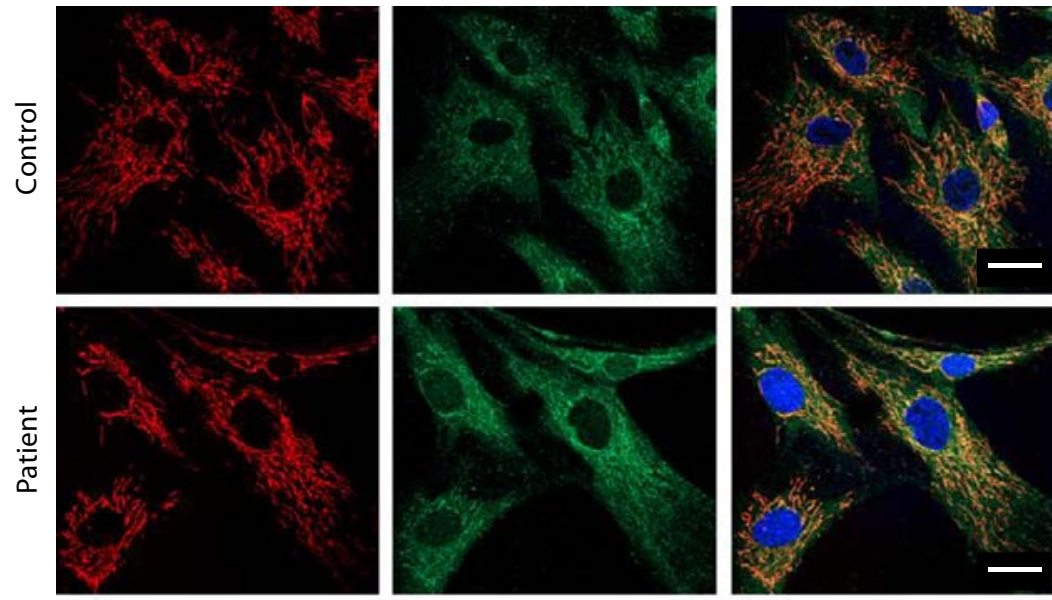

ATP synthase

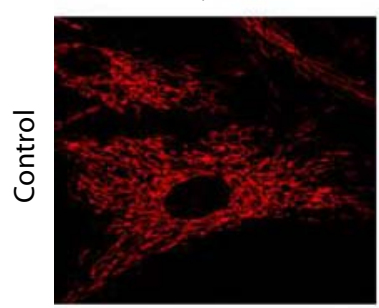

OPA3 - var. 2

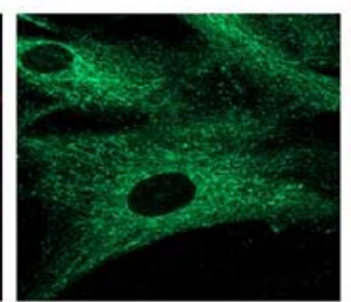

Merge + Hoechst
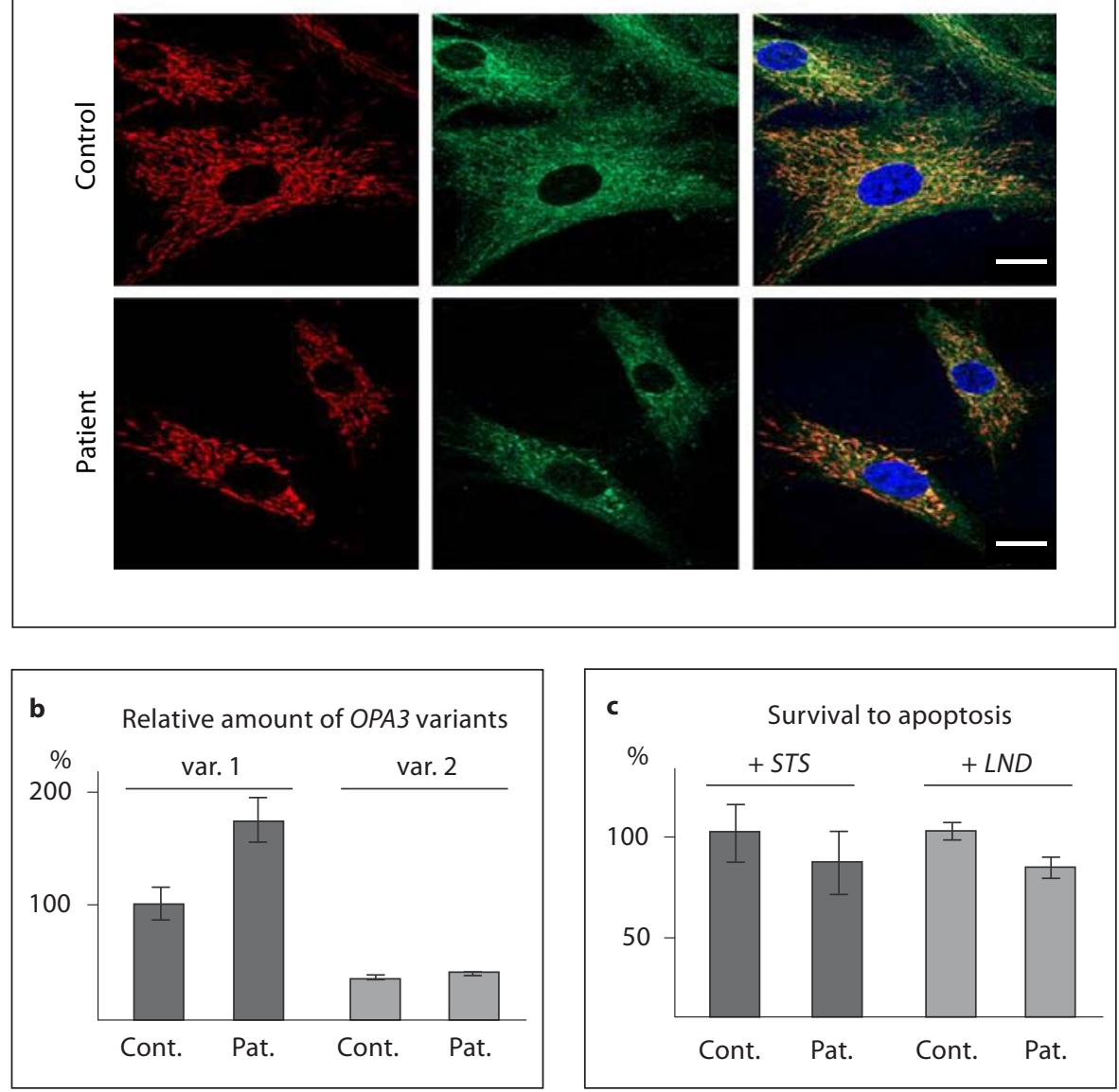
This is, to the best of our knowledge, the third report of a family harboring a dominant mutation in OPA3. Similarly to what was observed in one previously reported family [3], this OPA3 mutation is responsible for dominantly inherited optic atrophy and cataract (ADOAC) associated with multisystem neurologic disorder, including pes cavus, cerebellar ataxia, and areflexia.

Occurrence of pes cavus, cerebellar ataxia, and areflexia, evoking a 'Friedreich-like' phenotype, has already been reported in other mitochondriopathies such as disorders caused by mutations in OPA1, POLG1 [5], or in Leber hereditary optic neuropathy, suggesting a possible common physiopathological mechanism. The severe digestive dysfunction, present in the index case and her mother, seemed to be part of the extraneurological symptoms caused by $O P A 3$ mutation.
In conclusion, although mutations in $O P A 3$ are rare in patients with inherited optic atrophy, this report confirms that dominant mutations in OPA3 can be responsible for optic atrophy associated with severe neurological involvement.

\section{Acknowledgements}

We are indebted to the support of the Association Française contre les Myopathies, Retina France and Union Nationale des Aveugles et Déficients Visuels, and to the MRI platform.

\section{Disclosure Statement}

The authors have no conflicts of interest to disclose.

\section{References}

-1 Amati-Bonneau P, Valentino ML, Reynier P, et al: OPA1 mutations induce mitochondrial DNA instability and optic atrophy 'plus' phenotypes. Brain 2008;131:338-351.

- 2 Yu-Wai-Man P, Griffiths PG, Gorman GS, et al: Multi-system neurological disease is common in patients with OPA1 mutations. Brain 2010;133:771-786.

3 Reynier P, Amati-Bonneau P, Verny C, et al: $O P A 3$ gene mutations responsible for autosomal dominant optic atrophy and cataract. J Med Genet 2004;41:e110.

4 Neas K, Bennetts B, Carpenter K, et al: OPA3 mutation screening in patients with unexplained 3-methylglutaconic aciduria. J Inherit Metab Dis 2005;28:525-532.

5 Milone M, Benarroch EE, Wong LJ: POLGrelated disorders: defects of the nuclear and mitochondrial genome interaction. Neurology 2011;77:1847-1852. 